\title{
AUTOMORPHISM GROUPS OF AFFINE VARIETIES AND A CHARACTERIZATION OF AFFINE $n$-SPACE
}

\author{
HANSPETER KRAFT
}

\author{
Dedicated to Ernest Vinberg \\ at the occasion of his 80th birthday.
}

\begin{abstract}
We show that the automorphism group of affine $n$-space $\mathbb{A}^{n}$ determines $\mathbb{A}^{n}$ up to isomorphism: If $X$ is a connected affine variety such that $\operatorname{Aut}(X) \simeq \operatorname{Aut}\left(\mathbb{A}^{n}\right)$ as ind-groups, then $X \simeq \mathbb{A}^{n}$ as varieties.

We also show that every torus appears as $\operatorname{Aut}(X)$ for a suitable irreducible affine variety $X$, but that $\operatorname{Aut}(X)$ cannot be isomorphic to a semisimple group. In fact, if $\operatorname{Aut}(X)$ is finite dimensional and if $X \nsucceq \mathbb{A}^{1}$, then the connected component $\operatorname{Aut}(X)^{\circ}$ is a torus.

Concerning the structure of $\operatorname{Aut}\left(\mathbb{A}^{n}\right)$ we prove that any homomorphism $\operatorname{Aut}\left(\mathbb{A}^{n}\right) \rightarrow \mathcal{G}$ of ind-groups either factors through jac: Aut $\left(\mathbb{A}^{n}\right) \rightarrow \mathbb{k}^{*}$ where jac is the Jacobian determinant, or it is a closed immersion. For $\operatorname{SAut}\left(\mathbb{A}^{n}\right):=$ $\operatorname{ker}(\mathrm{jac}) \subseteq \operatorname{Aut}\left(\mathbb{A}^{n}\right)$ we show that every nontrivial homomorphism SAut $\left(\mathbb{A}^{n}\right) \rightarrow$ $\mathcal{G}$ is a closed immersion.

Finally, we prove that every non-trivial homomorphism $\varphi: \operatorname{SAut}\left(\mathbb{A}^{n}\right) \rightarrow$ $\operatorname{SAut}\left(\mathbb{A}^{n}\right)$ is an automorphism, and that $\varphi$ is given by conjugation with an element from $\operatorname{Aut}\left(\mathbb{A}^{n}\right)$.
\end{abstract}

\section{Introduction AND MAIN RESUlts}

Our base field $\mathbb{k}$ is algebraically closed of characteristic zero. For an affine variety $X$ the automorphism group $\operatorname{Aut}(X)$ has the structure of an affine ind-group. We will shortly recall the basic definitions in the following section 2 . The classical example is $\operatorname{Aut}\left(\mathbb{A}^{n}\right)$, the group of automorphisms of affine $n$-space $\mathbb{A}^{n}=\mathbb{k}^{n}$.

A fundamental question is how much information about $X$ can be retrieved from $\operatorname{Aut}(X)$. E.g. JelOnEK shows in [Jel15] that if $\operatorname{Aut}(X)$ is infinite, then $X$ is uniruled. Our main result shows that $\mathbb{A}^{n}$ is completely determined by its automorphism group.

Theorem 1.1. Let $X$ be a connected affine variety. If $\operatorname{Aut}(X) \simeq \operatorname{Aut}\left(\mathbb{A}^{n}\right)$ as ind-groups, then $X \simeq \mathbb{A}^{n}$ as varieties.

It is clear that $X$ has to be connected since the automorphism group does not change if we form the disjoint union of $\mathbb{A}^{n}$ with a variety $Y$ with trivial automorphism group. Some generalization of this result can be found in [Reg17].

Date: February 10, 2017; a version from October 8, 2014 is on arXiv 1501.06362 [math.RT].

2010 Mathematics Subject Classification. 20Gxx, 14Lxx, 14Rxx, 17Bxx, 20Fxx.

Key words and phrases. automorphism groups of affine varieties, ind-groups, Lie algebras of ind-groups, vector fields, affine $n$-space.

The author was partially supported by Swiss National Science Foundation. 
The proof of the theorem will follow from a more general result (Theorem 5.5, see Remark 5.4) where the group $\operatorname{Aut}\left(\mathbb{A}^{n}\right)$ is replaced by the subgroup $\mathcal{U}\left(\mathbb{A}^{n}\right)$ generated by the unipotent elements.

Another important question is which groups appear as automorphism groups of affine varieties. For finite groups we have the following result due to JELONEK.

Theorem 1.2 ([Jel15], Proposition 7.2). For every finite group $G$ and every $n \geq 1$ there is a n-dimensional smooth connected affine variety $X$ such that $\operatorname{Aut}(X) \simeq G$.

Moreover, there exist surfaces with infinite discrete automorphism groups (see [FK17, Proposition 12.7.1]). As for algebraic groups, we have Aut $\left(\mathbb{A}^{1}\right)=\operatorname{Aff}{ }_{1}$, and we will give examples where $\operatorname{Aut}(X)$ is a torus (Example 7.4). But other groups cannot appear as the next result shows.

Theorem 1.3. Let $X$ be a connected affine variety. If $\operatorname{dim} \operatorname{Aut}(X)<\infty$, then either $X \simeq \mathbb{A}^{1}$ or the connected component $\operatorname{Aut}(X)^{\circ}$ is a torus.

The last results concern the automorphism group $\operatorname{Aut}\left(\mathbb{A}^{n}\right)$ of affine $n$-space. This group has a closed normal subgroup $\operatorname{SAut}\left(\mathbb{A}^{n}\right)$ consisting of those automorphism $\mathbf{f}=\left(f_{1}, \ldots, f_{n}\right)$ whose Jacobian determinant $\operatorname{jac}(\mathbf{f}):=\operatorname{det}\left(\frac{\partial f_{i}}{\partial x_{j}}\right)_{(i, j)}$ is equal to $1:$

$$
\operatorname{SAut}\left(\mathbb{A}^{n}\right):=\operatorname{ker}\left(\operatorname{jac}: \operatorname{Aut}\left(\mathbb{A}^{n}\right) \rightarrow \mathbb{k}^{*}\right) .
$$

For an ind-group $\mathcal{G}$ the tangent space $T_{e} \mathcal{G}$ carries a canonical structure of a Liealgebra which we denote by Lie $\mathcal{G}$. For $\operatorname{SAut}\left(\mathbb{A}^{n}\right)$, the Lie algebra can be identified with $\operatorname{Vec}^{0}\left(\mathbb{A}^{n}\right)$, the vector fields $\xi$ on $\mathbb{A}^{n}$ with divergence $\operatorname{div} \xi=0$. This Lie algebra is simple, so one could expect that $\operatorname{SAut}\left(\mathbb{A}^{n}\right)$ is simple as an ind-group. This is claimed in [Sha66, Sha81], but the proofs turned out to be not correct (see [FK17, section 15]). What we can show here is the following.

Theorem 1.4. Let $n \geq 2$.

(1) Let $\varphi: \operatorname{Aut}\left(\mathbb{A}^{n}\right) \rightarrow \mathcal{G}$ be a homomorphism of ind-groups. Then either $\varphi$ factors through jac: $\operatorname{Aut}\left(\mathbb{A}^{n}\right) \rightarrow \mathbb{k}^{*}$, or $\varphi$ is a closed immersion, i.e. the image is closed and isomorphic to $\operatorname{Aut}\left(\mathbb{A}^{n}\right)$ under $\varphi$.

(2) Every nontrivial homomorphism $\operatorname{SAut}\left(\mathbb{A}^{n}\right) \rightarrow \mathcal{G}$ of ind-groups is a closed immersion.

This theorem has the following interesting applications. By definition, a representation of an ind-group $\mathcal{G}$ on a vector space $V$ of countable dimension is a homomorphism $\mathcal{G} \rightarrow \mathrm{GL}(V)$ such that the corresponding map $\mathcal{G} \times V \rightarrow V$ is a morphism of ind-varieties (see section 3). An action of an ind-group $\mathcal{G}$ on an affine variety $X$ is a homomorphism $\mathcal{G} \rightarrow \operatorname{Aut}(X)$ of ind-groups.

Corollary 1.5. Assume that $n \geq 2$.

(1) The ind-group $\operatorname{SAut}\left(\mathbb{A}^{n}\right)$ does not have a non-trivial finite dimensional representation.

(2) Assume that $\operatorname{SAut}\left(\mathbb{A}^{n}\right)$ acts nontrivially on a connected affine variety $X$. Then the action is faithful, and there are no fixed points.

Proof. (1) Let $\rho: \operatorname{SAut}\left(\mathbb{A}^{n}\right) \rightarrow \mathrm{GL}(V)$ be a finite dimensional representation. If $\rho$ is nontrivial, then it is a closed immersion, by Theorem 1.4(2). This is impossible, because $\mathrm{GL}(V)$ is finite dimensional. 
(2) We have a nontrivial homomorphism $\varphi: \operatorname{SAut}\left(\mathbb{A}^{n}\right) \rightarrow \operatorname{Aut}(X)$ which is a closed immersion, by Theorem 1.4(2). Thus the action is faithful, and the same is true for the induced action of $\mathrm{SL}_{n} \subseteq \operatorname{SAut}\left(\mathbb{A}^{n}\right)$. Since $X$ is connected, it follows that $\mathrm{SL}_{n}$ acts nontrivially on every irreducible component of $X$. This implies that for every fixed point $x \in X^{\mathrm{SL}_{n}}$ the tangent representation of $\mathrm{SL}_{n}$ on $T_{x} X$ is non-trivial. Hence, the tangent representation of $\operatorname{SAut}\left(\mathbb{A}^{n}\right)$ on every fixed point of $\operatorname{SAut}\left(\mathbb{A}^{n}\right)$ is also nontrivial, contradicting (1).

It is shown in [BKY12] that every automorphism of the ind-group $\operatorname{Aut}\left(\mathbb{A}^{n}\right)$ is inner, i.e. given by conjugation with a suitable $\mathrm{g} \in \operatorname{Aut}\left(\mathbb{A}^{n}\right)$ (cf. $[\mathrm{FK} 17$, Theorem 12.5.2]). This can be generalized in the following way.

\section{Theorem 1.6.}

(1) Every injective homomorphism $\varphi: \operatorname{Aut}\left(\mathbb{A}^{n}\right) \rightarrow \operatorname{Aut}\left(\mathbb{A}^{n}\right)$ is an isomorphism, and $\varphi=\operatorname{Int} \mathbf{g}$ for a well-defined $\mathbf{g} \in \operatorname{Aut}\left(\mathbb{A}^{n}\right)$.

(2) Every nontrivial homomorphism $\varphi: \operatorname{SAut}\left(\mathbb{A}^{n}\right) \rightarrow \operatorname{SAut}\left(\mathbb{A}^{n}\right)$ is an isomorphism, and $\varphi=\operatorname{Int} \mathbf{g}$ for a well-defined $\mathbf{g} \in \operatorname{Aut}\left(\mathbb{A}^{n}\right)$.

Remark 1.7. The analogue of Theorem 1.6 for vector fields, namely that every injective homomorphism $\varphi: \operatorname{Vec}\left(\mathbb{A}^{n}\right) \rightarrow \operatorname{Vec}\left(\mathbb{A}^{n}\right)$ of Lie algebras is an automorphism, would imply the Jacobian Conjecture in dimension $n$, see [KR17, Corollary 4.4].

We finally mention the following example showing that bijective homomorphisms of ind-groups are not necessarily isomorphisms. The details can be found in [FK17, section 8], cf. [BW00, section 11, last paragraph]. Denote by $\mathbb{k}\langle x, y\rangle$ the free associative $\mathbb{k}$-algebra in two generators. Then $\operatorname{Aut}(\mathbb{k}\langle x, y\rangle)$ is an ind-group, and we have a canonical homomorphism $\pi: \operatorname{Aut}(\mathbb{k}\langle x, y\rangle) \rightarrow \operatorname{Aut}(\mathbb{k}[x, y])$.

Proposition 1.8. The map $\pi: \operatorname{Aut}(\mathbb{k}\langle x, y\rangle) \rightarrow \operatorname{Aut}(\mathbb{k}[x, y])$ is a bijective homomorphism of ind-groups, but it is not an isomorphism, because it is not an isomorphism on the Lie algebras.

Note that $\operatorname{Aut}(\mathbb{k}\langle x, y\rangle)$ is generated by the closed algebraic subgroups $G \subseteq$ $\operatorname{Aut}(\mathbb{k}\langle x, y\rangle)$, and that $\pi: G \stackrel{\sim}{\rightarrow} \pi(G)$ is an isomorphism for these subgroups.

\section{NOTATION AND PRELIMINARY RESULTS}

The notion of an ind-group goes back to Shafarevich who called these objects infinite dimensional groups, see [Sha66, Sha81]. We refer to [Kum02] and the notes [FK17] for basic notation in this context.

Definition 2.1. An ind-variety $\mathcal{V}$ is a set together with an ascending filtration $\mathcal{V}_{0} \subseteq \mathcal{V}_{1} \subseteq \mathcal{V}_{2} \subseteq \cdots \subseteq \mathcal{V}$ such that the following holds:

(1) $\mathcal{V}=\bigcup_{k \in \mathbb{N}} \mathcal{V}_{k}$

(2) Each $\mathcal{V}_{k}$ has the structure of an algebraic variety;

(3) For all $k \in \mathbb{N}$ the inclusion $\mathcal{V}_{k} \hookrightarrow \mathcal{V}_{k+1}$ is closed immersion.

A morphism between ind-varieties $\mathcal{V}=\bigcup_{k} \mathcal{V}_{k}$ and $\mathcal{W}=\bigcup_{m} \mathcal{W}_{m}$ is a map $\varphi: \mathcal{V} \rightarrow$ $\mathcal{W}$ such that, for every $k$, there is an $m$ with the properties that $\varphi\left(\mathcal{V}_{k}\right) \subseteq \mathcal{W}_{m}$ and that the induced map $\mathcal{V}_{k} \rightarrow \mathcal{W}_{m}$ is a morphism of varieties. Isomorphisms of indvarieties are defined in the usual way.

Two filtrations $\mathcal{V}=\bigcup_{k \in \mathbb{N}} \mathcal{V}_{k}$ and $\mathcal{V}=\bigcup_{k \in \mathbb{N}} \mathcal{V}_{k}^{\prime}$ are called equivalent if, for any $k$, there is an $m$ such that $\mathcal{V}_{k} \subseteq \mathcal{V}_{m}^{\prime}$ is a closed subvariety as well as $\mathcal{V}_{k}^{\prime} \subseteq \mathcal{V}_{m}$. 
Equivalently, the identity map id: $\mathcal{V}=\bigcup_{k \in \mathbb{N}} \mathcal{V}_{k} \rightarrow \mathcal{V}=\bigcup_{k \in \mathbb{N}} \mathcal{V}_{k}^{\prime}$ is an isomorphism of ind-varieties.

An ind-variety $\mathcal{V}$ has a natural topology where $S \subseteq \mathcal{V}$ is open, resp. closed, if $S_{k}:=S \cap \mathcal{V}_{k} \subseteq \mathcal{V}_{k}$ is open, resp. closed, for all $k$. Obviously, a locally closed subset $S \subseteq \mathcal{V}$ has a natural structure of an ind-variety. It is called an ind-subvariety. An ind-variety $\mathcal{V}$ is called affine if all $\mathcal{V}_{k}$ are affine. A subset $X \subseteq \mathcal{V}$ is called algebraic if it is locally closed and contained in some $\mathcal{V}_{k}$. Such an $X$ has a natural structure of an algebraic variety.

Example 2.2. (1) Any $\mathbb{k}$-vector space $V$ of countable dimension carries the structure of an (affine) ind-variety by choosing an increasing sequence of finite dimensional subspaces $V_{k}$ such that $V=\bigcup_{k} V_{k}$. Clearly, all these filtrations are equivalent.

(2) If $R$ is a commutative $\mathbb{k}$-algebra of countable dimension, $\mathfrak{a} \subseteq R$ a subspace, e.g. an ideal, and $S \subseteq \mathbb{k}\left[x_{1}, \ldots, x_{n}\right]$ a set of polynomials, then the subset

$$
\left\{\left(a_{1}, \ldots, a_{n}\right) \in R^{n} \mid f\left(a_{1}, \ldots, a_{n}\right) \in \mathfrak{a} \text { for all } f \in S\right\} \subseteq R^{n}
$$

is a closed ind-subvariety of $R^{n}$.

For any ind-variety $\mathcal{V}=\bigcup_{k \in \mathbb{N}} \mathcal{V}_{k}$ we can define the tangent space in $x \in \mathcal{V}$ in the obvious way. We have $x \in \mathcal{V}_{k}$ for $k \geq k_{0}$, and $T_{x} \mathcal{V}_{k} \subseteq T_{x} \mathcal{V}_{k+1}$ for $k \geq k_{0}$, and then define

$$
T_{x} \mathcal{V}:=\underset{k \geq k_{0}}{\lim _{k}} T_{x} \mathcal{V}_{k}
$$

which is a vector space of countable dimension. A morphism $\varphi: \mathcal{V} \rightarrow \mathcal{W}$ induces linear maps $d \varphi_{x}: T_{x} \mathcal{V} \rightarrow T_{\varphi(x)} \mathcal{W}$ for every $x \in X$. Clearly, for a $\mathbb{k}$-vector space $V$ of countable dimension and for any $v \in V$ we have $T_{v} V=V$ in a canonical way.

The product of two ind-varieties is defined in the obvious way. This allows to define an ind-group as an ind-variety $\mathcal{G}$ with a group structure such that multiplication $\mathcal{G} \times \mathcal{G} \rightarrow \mathcal{G}:(g, h) \mapsto g \cdot h$, and inverse $\mathcal{G} \rightarrow \mathcal{G}: g \mapsto g^{-1}$, are both morphisms.

Remark 2.3. Let $G \subseteq \mathcal{G}$ be a subgroup. If $G$ is an algebraic subset, i.e. locally closed and contained in $\mathcal{G}_{k}$ for some $k$, then $G$ is an algebraic group and is closed in $\mathcal{G}$. We will call such a $G$ an algebraic subgroup.

Conversely, if $G$ is an algebraic group and $\varphi: G \rightarrow \mathcal{G}$ a homomorphism of indgroups, then $\varphi(G) \subseteq \mathcal{G}$ is a closed subgroup and an algebraic subset. The easy proofs are left to the reader.

If $\mathcal{G}$ is an affine ind-group, then $T_{e} \mathcal{G}$ has a natural structure of a Lie algebra which will be denoted by Lie $\mathcal{G}$. The structure is obtained by showing that every $A \in T_{e} \mathcal{G}$ defines a unique left-invariant vector field $\delta_{A}$ on $\mathcal{G}$, see [Kum02, Proposition 4.2.2, p. 114].

Definition 2.4. An ind-group $\mathcal{G}=\bigcup_{k} \mathcal{G}_{k}$ is called discrete if $\mathcal{G}_{k}$ is finite for all $k$. Clearly, $\mathcal{G}$ is discrete if and only if Lie $\mathcal{G}$ is trivial.

The next result can be found in [FK17, sections 4.1 and 4.6]. Here $\operatorname{Vec}(X)$ denotes the Lie algebra of (algebraic) vector fields on $X$, i.e. $\operatorname{Vec}(X)=\operatorname{Der}(\mathcal{O}(X))$, the Lie algebra of derivations of $\mathcal{O}(X)$.

Proposition 2.5. Let $X$ be an affine variety. Then $\operatorname{Aut}(X)$ has a natural structure of an affine ind-group, and there is a canonical embedding $\xi: \operatorname{Lie} \operatorname{Aut}(X) \hookrightarrow \operatorname{Vec}(X)$ of Lie algebras. 
Remark 2.6. For $X=\mathbb{A}^{n}$ the embedding $\xi$ identifies Lie $\left.\operatorname{Aut}\left(\mathbb{A}^{n}\right)\right)$ with $\operatorname{Vec}{ }^{\mathrm{c}}\left(\mathbb{A}^{n}\right)$, the vector fields $\delta=\sum_{i} f_{i} \frac{\partial}{\partial x_{i}}$ with constant divergence: $\operatorname{div} \delta:=\sum_{i} \frac{\partial f_{i}}{\partial x_{i}} \in \mathbb{k}$, see [FK17, Proposition 4.9.1].

The Jacobian determinant $\operatorname{jac}(\mathbf{f}):=\operatorname{det}\left(\frac{\partial f_{i}}{\partial x_{j}}\right)_{(i, j)}$ of an automorphism $\mathbf{f}=$ $\left(f_{1}, \ldots, f_{n}\right)$ of $\mathbb{A}^{n}$ defines a homomorphism jac: Aut $\left(\mathbb{A}^{n}\right) \rightarrow \mathbb{k}^{*}$ of ind-groups. Setting $\operatorname{SAut}\left(\mathbb{A}^{n}\right):=\operatorname{ker}$ jac one sees that $\xi$ identifies Lie SAut $\left(\mathbb{A}^{n}\right)$ with $\operatorname{Vec}\left(\mathbb{A}^{n}\right)$, the vector fields $\delta$ with $\operatorname{div} \delta=0$, see [FK17, Remark 4.9.3].

It is known that for $n \geq 2$ the Lie algebra Lie $\operatorname{SAut}\left(\mathbb{A}^{n}\right)$ is simple and that $\operatorname{Lie} \operatorname{SAut}\left(\mathbb{A}^{n}\right) \subseteq \operatorname{Lie} \operatorname{Aut}\left(\mathbb{A}^{n}\right)$ is the only proper ideal, see [Sha81, Lemma 3]. Moreover, both Lie algebras are generated by the subalgebras Lie $G$ where $G$ is an algebraic subgroup.

Another result which we will need is proved in [FK17, Proposition 2.7.6].

Proposition 2.7. Let $\varphi, \psi: \mathcal{G} \rightarrow \mathcal{H}$ be two homomorphisms of ind-groups. Assume that $\mathcal{G}$ is connected and that $d \varphi_{e}=d \psi_{e}:$ Lie $\mathcal{G} \rightarrow \operatorname{Lie} \mathcal{H}$. Then $\varphi=\psi$.

A final result which we will use can be found in [KRZ17]. Denote by $\operatorname{Aff}_{n} \subseteq$ $\operatorname{Aut}\left(\mathbb{A}^{n}\right)$ the subgroup of affine transformations, i.e. Aff ${ }_{n}=\mathrm{GL}_{n}(\mathbb{k}) \ltimes\left(\mathbb{k}^{n}\right)^{+}$. Similarly, the subgroup $\mathrm{SAff}_{n} \subseteq \mathrm{Aff}_{n}$ consists of the affine transformations with determinant 1, i.e. $\mathrm{SAff}_{n}=\mathrm{SL}_{n}(\mathbb{k}) \ltimes\left(\mathbb{k}^{n}\right)^{+}$.

Proposition 2.8. Let $X$ be a connected affine variety with a faithful action of SAff $_{n}$. If $\operatorname{dim} X \leq n$, then $X$ is $\mathrm{SAff}_{n}$-isomorphic to $\mathbb{A}^{n}$.

Remark 2.9. It is shown in [KRZ17] that the same holds if we replace $\mathrm{SAff}_{n}$ by $\operatorname{Aff}_{n}$. Using Theorem 1.6 we see that we can replace $\operatorname{SAff}_{n}$ by $\operatorname{Aut}\left(\mathbb{A}^{n}\right)$ or $\operatorname{SAut}\left(\mathbb{A}^{n}\right)$ as well.

\section{The ADJOINT REPRESENTATION}

Following [Kum02, section 4.2] we define a representation of an ind-group $\mathcal{G}$ on a vector space $V$ of countable dimension to be a homomorphism $\rho: \mathcal{G} \rightarrow \operatorname{GL}(V)$ of groups such that the induced map $\mathcal{G} \times V \rightarrow V$ is a morphism of ind-varieties. Note that $\operatorname{GL}(V)$ does not have the structure of an ind-variety if $\operatorname{dim} V=\infty$. However, if $L$ is a finitely generated Lie algebra, then $\operatorname{Aut}_{\text {Lie }}(L)$ has a natural structure of an ind-group which is defined in the following way (see [FK17, section 7] where we define an ind-group structure on $\operatorname{Aut}(R)$ for any finitely generated general algebra $R$, i.e. a $\mathbb{k}$-vector space $R$ endowed with a bilinear map $R \times R \rightarrow R$ ).

Choose a finite-dimensional subspace $L_{0} \subseteq L$ which generates $L$ as a Lie algebra. Then the restriction map $\operatorname{End}_{\text {Lie }}(L) \rightarrow \operatorname{Hom}\left(L_{0}, L\right)$ is injective and the image is a closed affine ind-subvariety. (To see this write $L$ as the quotient of the free Lie algebra $F\left(L_{0}\right)$ over $L_{0}$ modulo an ideal $I$.) Choosing a filtration $L=\bigcup_{k>0} L_{k}$ by finite-dimensional subspaces, we set $\operatorname{End}_{\text {Lie }}(L)_{k}:=\left\{\alpha \in \operatorname{End}_{\text {Lie }}(L) \mid \alpha\left(L_{0}\right) \subseteq L_{k}\right\}$ which is a closed subvariety of $\operatorname{Hom}\left(L_{0}, L_{k}\right)$ (see Example 2.2). Then we define the ind-structure on $\operatorname{Aut}_{\text {Lie }}(L)$ by identifying $\operatorname{Aut}_{\text {Lie }}(L)$ with the closed subset

$\left\{(\alpha, \beta) \in \operatorname{End}_{\text {Lie }}(L) \times \operatorname{End}_{\text {Lie }}(L) \mid \alpha \circ \beta=\beta \circ \alpha=\operatorname{id}_{L}\right\} \subseteq \operatorname{End}_{\text {Lie }}(L) \times \operatorname{End}_{\text {Lie }}(L)$, i.e.

$$
\operatorname{Aut}_{\text {Lie }}(L)_{k}:=\left\{\alpha \in \operatorname{Aut}_{\text {Lie }}(L) \mid \alpha, \alpha^{-1} \in \operatorname{End}_{\text {Lie }}(L)_{k}\right\}
$$


It follows that $\operatorname{Aut}_{\text {Lie }}(L)$ is an affine ind-group with the usual functorial properties. In particular, we have the following result.

Lemma 3.1. Let $\mathcal{G}$ be an ind-group, and let $\rho: \mathcal{G} \rightarrow \operatorname{Aut}_{\text {Lie }}(L)$ be an abstract homomorphism where $L$ is a finitely generated Lie algebra. Then $\rho$ is a homomorphism of ind-groups if and only if $\rho$ is a representation, i.e. the map $\rho: \mathcal{G} \times L \rightarrow L$ is a morphism of ind-varieties.

Proof. Assume that $L$ is generated by the finite dimensional subspace $L_{0} \subseteq L$. If $\mathcal{G}=\bigcup_{j} \mathcal{G}_{j}$ and if $\rho: \mathcal{G} \times L \rightarrow L$ is a morphism, then, for any $j$, there is a $k=k(j)$ such that $\rho\left(\mathcal{G}_{j} \times L_{0}\right) \subseteq L_{k}$ and $\rho\left(\mathcal{G}_{j}^{-1} \times L_{0}\right) \subseteq L_{k}$. Hence, $\rho\left(\mathcal{G}_{j}\right) \subseteq \operatorname{Aut}_{\text {Lie }}(L)_{k}$, and the map $\mathcal{G}_{j} \rightarrow \operatorname{Hom}\left(L_{0}, L_{k}\right)$ is clearly a morphism.

Now assume that $\mathcal{G} \rightarrow \operatorname{Aut}_{\text {Lie }}(L)$ is a homomorphism of ind-groups. Then, for any $j$, there is a $k=k(j)$ such that $\rho\left(\mathcal{G}_{j}\right) \subseteq \operatorname{Aut}_{\text {Lie }}(L)_{k} \hookrightarrow \operatorname{Hom}\left(L_{0}, L_{k}\right)$. Hence, $\rho\left(\mathcal{G}_{j} \times L_{0}\right) \subseteq L_{k}$, and $\mathcal{G}_{j} \times L_{0} \rightarrow L_{k}$ is a morphism.

The adjoint representation $\mathrm{Ad}: \mathcal{G} \rightarrow \operatorname{Aut}_{\text {Lie }}($ Lie $\mathcal{G})$ of an ind-group $\mathcal{G}$ is defined in the usual way: $\operatorname{Ad} g:=(d \operatorname{Int} g)_{e}: \operatorname{Lie} \mathcal{G} \stackrel{\sim}{\rightarrow}$ Lie $\mathcal{G}$ where $\operatorname{Int} g$ is the inner automorphism $h \mapsto g h g^{-1}$.

Proposition 3.2. For any ind-group $\mathcal{G}$ the canonical map Ad: $\mathcal{G} \rightarrow \operatorname{Aut}_{\text {Lie }}(\operatorname{Lie} \mathcal{G})$ is a homomorphism of ind-groups.

Proof. Let $\gamma: \mathcal{G} \times \mathcal{G} \rightarrow \mathcal{G}$ denote the morphism $(g, h) \mapsto g h g^{-1}$. For any $g \in \mathcal{G}$, the map $\gamma_{g}: \mathcal{G} \rightarrow \mathcal{G}, h \mapsto g h g^{-1}$, is an isomorphism of ind-groups, and its differential $\operatorname{Ad}(g)=\left(d \gamma_{g}\right)_{e}:$ Lie $\mathcal{G} \rightarrow$ Lie $\mathcal{G}$ is an isomorphism of Lie algebras. If $\mathcal{G}=\bigcup_{k} \mathcal{G}_{k}$, then for any $p, q \in \mathbb{N}$ there is an $m \in \mathbb{N}$ such that $\gamma: \mathcal{G}_{p} \times \mathcal{G}_{p} \rightarrow \mathcal{G}_{m}$. Clearly, for $g \in$ $\mathcal{G}_{p}, \operatorname{Ad} g$ is given by $\left(d \gamma_{g}\right)_{e}: T_{e} \mathcal{G}_{q} \rightarrow T_{e} \mathcal{G}_{m}$, and the map $\mathcal{G}_{k} \rightarrow \operatorname{Hom}\left(T_{e} \mathcal{G}_{q}, T_{e} \mathcal{G}_{m}\right)$ is a morphism, by the following lemma. Now the claim follows from Lemma 3.1 above.

Lemma 3.3. Let $\Phi: X \times Y \rightarrow Z$ be a morphism of affine varieties and set $\Phi_{x}(y):=$ $\Phi(x, y)$. Assume that there exist $y_{0} \in Y$ and $z_{0} \in Z$ such that $\Phi_{x}\left(y_{0}\right)=z_{0}$ for all $x \in X$. Then the induced map $X \rightarrow \operatorname{Hom}\left(T_{y_{0}} Y, T_{z_{0}} Z\right), x \mapsto d_{y_{0}} \Phi_{x}$, is a morphism.

Proof. We can assume that $Y, Z$ are vector spaces, $Y=W$ and $Z=V$. Choose bases $\left(w_{1}, \ldots, w_{m}\right)$ of $W$ and $\left(v_{1}, \ldots, v_{n}\right)$ of $V$. Then $\Phi$ is given by an element of the form

$$
\sum_{i=1}^{n} \sum_{j} f_{i j} \otimes h_{i j} \otimes v_{i}, \text { where } f_{i j} \in \mathcal{O}(X) \text { and } h_{i j} \in \mathcal{O}(Y)=\mathbb{k}\left[y_{1}, \ldots, y_{m}\right],
$$

and so the differential $\left(d \Phi_{x}\right)_{y_{0}}: W \rightarrow V$ is given by the matrix

$$
\left(\sum_{j} f_{i j}(x) \frac{\partial h_{i j}}{\partial y_{k}}\left(y_{0}\right)\right)_{(i, k)}
$$

whose entries are regular functions on $x$. The claim follows.

We have shown in [KR17] that the adjoint representation

$$
\operatorname{Ad}_{\operatorname{Aut}\left(\mathbb{A}^{n}\right)}: \operatorname{Aut}\left(\mathbb{A}^{n}\right) \stackrel{\sim}{\rightarrow} \operatorname{Aut}_{\text {Lie }}\left(\operatorname{Lie} \operatorname{Aut}\left(\mathbb{A}^{n}\right)\right)
$$

and the induced homomorphism $\rho: \operatorname{Aut}_{\text {Lie }}\left(\operatorname{Lie} \operatorname{Aut}\left(\mathbb{A}^{n}\right)\right) \stackrel{\sim}{\longrightarrow} \operatorname{Aut}_{\text {Lie }}\left(\operatorname{Lie} \operatorname{SAut}\left(\mathbb{A}^{n}\right)\right)$ are both bijective. They are also homomorphisms of ind-groups: For $\operatorname{Ad}_{\operatorname{Aut}\left(\mathbb{A}^{n}\right)}$ this 
is Proposition 3.2 above, and for $\rho$ it is obvious. But this does not necessarily imply that the maps are isomorphisms of ind-groups, see Proposition 1.8. However, for $\operatorname{Aut}\left(\mathbb{A}^{n}\right)$ it is true, and we will need this for the proof of Theorem 1.4 in the following section.

Proposition 3.4. The adjoint representation

$$
\operatorname{Ad}_{\operatorname{Aut}\left(\mathbb{A}^{n}\right)}: \operatorname{Aut}\left(\mathbb{A}^{n}\right) \stackrel{\sim}{\rightarrow} \operatorname{Aut}_{\text {Lie }}\left(\operatorname{Lie} \operatorname{Aut}\left(\mathbb{A}^{n}\right)\right)
$$

is an isomorphism of ind-groups.

Proof. We will use here the identification of Lie $\operatorname{Aut}\left(\mathbb{A}^{n}\right)$ with $\operatorname{Vec}{ }^{c}\left(\mathbb{A}^{n}\right)$, see Remark 2.6. Put $\partial_{x_{i}}:=\frac{\partial}{\partial x_{i}}$.

Let $\mathbf{f}=\left(f_{1}, \ldots, f_{n}\right) \in \operatorname{Aut}\left(\mathbb{A}^{n}\right)$ and set $\theta:=\operatorname{Ad}\left(\mathbf{f}^{-1}\right) \in \operatorname{Aut}_{\text {Lie }}\left(\operatorname{Vec}^{\mathrm{c}}\left(\mathbb{A}^{n}\right)\right)$. Then the matrix $\left(\theta\left(\partial_{x_{k}}\right) x_{j}\right)_{(j, k)}$ is invertible, and

$$
\left(\theta\left(\partial_{x_{k}}\right) x_{j}\right)_{(j, k)}^{-1}=\operatorname{Jac}(\mathbf{f})=\left(\frac{\partial f_{j}}{\partial x_{i}}\right)_{(i, j)},
$$

see [KR17, Remark 4.2]. We now claim that the map

$$
\theta \mapsto\left(\theta\left(\partial_{x_{k}}\right) x_{j}\right)_{(j, k)}^{-1}: \operatorname{Aut}_{\text {Lie }}\left(\operatorname{Vec}^{\mathrm{c}}\left(\mathbb{A}^{n}\right)\right) \rightarrow \mathrm{M}_{n}\left(\mathbb{k}\left[x_{1}, \ldots, x_{n}\right]\right)
$$

is a well-defined morphism of ind-varieties. In fact, $\theta \mapsto \theta\left(\partial_{x_{k}}\right) x_{j}$ is the composition of the orbit map $\theta \mapsto \theta\left(\partial_{x_{k}}\right): \operatorname{Aut}_{\text {Lie }}\left(\operatorname{Vec}^{\mathrm{c}}\left(\mathbb{A}^{n}\right)\right) \rightarrow \operatorname{Vec}^{\mathrm{c}}\left(\mathbb{A}^{n}\right)$ and the evaluation $\operatorname{map} \delta \mapsto \delta\left(x_{j}\right): \operatorname{Vec}^{\mathrm{c}}\left(\mathbb{A}^{n}\right) \rightarrow \mathbb{k}\left[x_{1}, \ldots, x_{n}\right]$, hence $\theta \mapsto \Theta:=\left(\theta\left(\partial_{x_{k}}\right) x_{j}\right)_{(j, k)}$ is a morphism. Since jac $(\Theta) \in \mathbb{k}^{*}$ the claim follows.

Now recall that the gradient $\mathbb{k}\left[x_{1}, \ldots, x_{n}\right] \rightarrow \mathbb{k}\left[x_{1}, \ldots, x_{n}\right]^{n}, f \mapsto\left(\frac{\partial f}{\partial x_{1}}, \ldots, \frac{\partial f}{\partial x_{n}}\right)$, defines an isomorphism

$$
\gamma: \mathbb{k}\left[x_{1}, \ldots, x_{n}\right]_{\geq 1} \stackrel{\sim}{\rightarrow} \Gamma:=\left\{\left(h_{1}, \ldots, h_{n}\right) \mid \frac{\partial h_{i}}{\partial x_{j}}=\frac{\partial h_{j}}{\partial x_{i}} \text { for all } i<j\right\} .
$$

It follows from $(*)$ that the rows of the matrix $\left(h_{i j}\right)_{(i, j)}:=\left(\theta\left(\partial_{x_{k}}\right) x_{j}\right)_{(j, k)}^{-1}$ belong to $\Gamma$, so that we get a morphism

$$
\psi: \operatorname{Aut}_{\text {Lie }}\left(\operatorname{Vec}^{\mathrm{c}}\left(\mathbb{A}^{n}\right)\right) \rightarrow \mathbb{k}\left[x_{1}, \ldots, x_{n}\right]^{n}, \theta \mapsto\left(f_{1}, \ldots, f_{n}\right),
$$

where $f_{i}:=\gamma^{-1}\left(h_{i 1}, \ldots, h_{i n}\right) \in \mathbb{k}\left[x_{1}, \ldots, x_{n}\right]_{\geq 1}$. By construction, we have

$$
\psi(\theta)=\psi\left(\operatorname{Ad}\left(\mathbf{f}^{-1}\right)\right)=\mathbf{f}_{0}:=\left(f_{1}-f_{1}(0), \ldots, f_{n}-f_{n}(0)\right)=\mathbf{t}_{-\mathbf{f}(0)} \circ \mathbf{f}
$$

where $\mathbf{t}_{a}$ is the translation $v \mapsto v+a$. Let $S \subseteq$ Aff $_{n}$ be the subgroup of translations, and set $\tilde{S}:=\operatorname{Ad}(S)$. Then $\tilde{S} \subseteq \operatorname{Aut}_{\text {Lie }}\left(\operatorname{Vec}^{\mathrm{c}}\left(\mathbb{A}^{n}\right)\right)$ is a closed algebraic subgroup and Ad $: S \rightarrow \tilde{S}$ is an isomorphism. It follows from $(* *)$ that $\operatorname{Ad}(\psi(\theta)) \cdot \theta=\operatorname{Ad}\left(\mathbf{t}_{-\mathbf{f}(0)}\right) \in$ $\tilde{S}$, and so

$$
\tilde{\psi}(\theta):=\psi(\theta)^{-1} \cdot\left(\left.\operatorname{Ad}\right|_{S}\right)^{-1}(\operatorname{Ad}(\psi(\theta)) \cdot \theta)
$$

is a well-defined morphism $\tilde{\psi}: \operatorname{Aut}_{\mathrm{Lie}}\left(\operatorname{Vec}^{\mathrm{c}}\left(\mathbb{A}^{n}\right)\right) \rightarrow \operatorname{Aut}\left(\mathbb{A}^{n}\right)$ with the property that

$$
\operatorname{Ad}(\tilde{\psi}(\theta))=\operatorname{Ad}\left(\psi(\theta)^{-1}\right) \cdot \operatorname{Ad}(\psi(\theta)) \cdot \theta=\theta .
$$

Thus $\operatorname{Ad}: \operatorname{Aut}\left(\mathbb{A}^{n}\right) \rightarrow \operatorname{Aut}_{\text {Lie }}\left(\operatorname{Lie} \operatorname{Aut}\left(\mathbb{A}^{n}\right)\right)$ is an isomorphism, with inverse $\tilde{\psi}$. 
Remark 3.5. Clearly, the restriction $\rho: \operatorname{Aut}$ Lie $\left(\operatorname{Lie} \operatorname{Aut}\left(\mathbb{A}^{n}\right)\right) \rightarrow \operatorname{Aut}_{\text {Lie }}\left(\operatorname{Lie} \operatorname{SAut}\left(\mathbb{A}^{n}\right)\right)$ is a homomorphism of ind-groups, and it is bijective, see [KR17]. It follows from (1) that the composition $\rho \circ \operatorname{Ad:} \operatorname{Aut}\left(\mathbb{A}^{n}\right) \rightarrow \operatorname{Aut}_{\text {Lie }}\left(\operatorname{Lie} \operatorname{SAut}\left(\mathbb{A}^{n}\right)\right)$ is a bijective homomorphism of ind-groups. Now we use Theorem 1.4(1) to conclude that $\rho \circ \mathrm{Ad}$ is an isomorphism, hence $\rho$ is an isomorphism, too.

\section{Proof of the Theorems 1.4 And 1.6}

Proof of Theorem 1.4. (1) Let $\varphi: \operatorname{Aut}\left(\mathbb{A}^{n}\right) \rightarrow \mathcal{G}$ be a homomorphism of ind-groups such that $d \varphi$ is injective. We can assume that $\mathcal{G}=\overline{\varphi\left(\operatorname{Aut}\left(\mathbb{A}^{n}\right)\right)}$, and we will show that $\varphi$ is an isomorphism. The basic idea is to construct a homomorphism $\psi: \mathcal{G} \rightarrow$ $\operatorname{Aut}\left(\mathbb{A}^{n}\right)$ such that $\psi \circ \varphi=\mathrm{id}$. By Proposition 4.1 below this implies that $\varphi$ is a closed immersion, hence an isomorphism.

Denote by $L \subseteq$ Lie $\mathcal{G}$ the image of $d \varphi$. For any $g \in \operatorname{Aut}\left(\mathbb{A}^{n}\right)$ we have $d \varphi \circ \operatorname{Ad}(g)=$ $\operatorname{Ad}(\varphi(g)) \circ d \varphi$. In particular, $L$ is stable under $\operatorname{Ad}(\varphi(g))$, hence stable under $\operatorname{Ad}(\mathcal{G})$, because $\varphi\left(\operatorname{Aut}\left(\mathbb{A}^{n}\right)\right)$ is dense in $\mathcal{G}$. Thus we get the following commutative diagram of homomorphisms of ind-groups

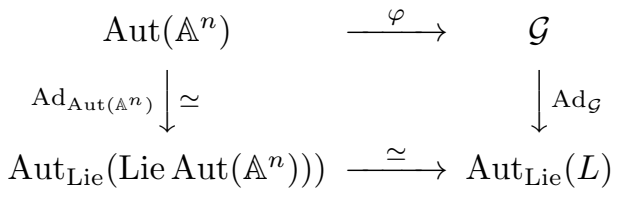

where the first vertical map is an isomorphism, by Proposition 3.4. Thus, the composition $\operatorname{Ad}_{\mathcal{G}} \circ \varphi: \operatorname{Aut}\left(\mathbb{A}^{n}\right) \rightarrow \operatorname{Aut}_{\text {Lie }}(L) \simeq \operatorname{Aut}\left(\mathbb{A}^{n}\right)$ is an isomorphism, and so $\varphi$ is also an isomorphism, by Proposition 4.1 below.

If $d \varphi$ is not injective, then $\operatorname{ker} d \varphi \supseteq \operatorname{LieSAut}\left(\mathbb{A}^{n}\right)(\operatorname{Remark} 2.6)$ and so $d \varphi=$ $f \circ d$ jac where $f: \mathbb{k} \rightarrow$ Lie $\mathcal{G}$ is a Lie algebra homomorphism. If $\mathbb{k}^{*} \subseteq \mathrm{GL}_{n}(\mathbb{k})$ denotes the center, then $\left.\varphi\right|_{\mathbb{k}^{*}}: \mathbb{k}^{*} \rightarrow \mathcal{G}$ factor through $?^{n}: \mathbb{k}^{*} \rightarrow \mathbb{k}^{*}$, because $\mathrm{SL}_{n}(\mathbb{k}) \subseteq \operatorname{ker} \varphi$, i.e. $\varphi(z)=\rho\left(z^{n}\right)$ for any $z \in \mathbb{k}^{*}$ and a suitable homomorphism $\rho: \mathbb{k}^{*} \rightarrow \mathcal{G}$ of indgroups. By construction, $d \rho_{e}=f: \mathbb{k} \rightarrow$ Lie $\mathcal{G}$, and so the two homomorphisms $\varphi$ and $\rho \circ$ jac have the same differential. Thus, by Proposition 2.7, we get $\varphi=\rho \circ$ jac, and we are done.

(2) Let $\varphi: \operatorname{SAut}\left(\mathbb{A}^{n}\right) \rightarrow \mathcal{G}$ be a homomorphism of ind-groups. If $d \varphi_{e}$ is not injective, then $d \varphi_{e}$ is the trivial map (Remark 2.6), hence $d \varphi_{e}=d \bar{\varphi}_{e}$ where $\bar{\varphi}: \mathbf{g} \mapsto$ $e$ is the constant homomorphism. Again by Proposition 2.7 we get $\varphi=\bar{\varphi}$.

If $d \varphi_{e}$ is injective, set $L:=d \varphi_{e}\left(\operatorname{Lie} \operatorname{SAut}\left(\mathbb{A}^{n}\right)\right) \subseteq \operatorname{Lie} \mathcal{G}$. As above we can assume that $\mathcal{G}=\overline{\varphi\left(\operatorname{SAut}\left(\mathbb{A}^{n}\right)\right)}$. Since $L$ is stable under $\operatorname{Ad} \varphi(\mathbf{g})$ for all $\mathbf{g} \in \operatorname{SAut}\left(\mathbb{A}^{n}\right)$ it is also stable under $\mathcal{G}$, and we get, as above, the following commutative diagram

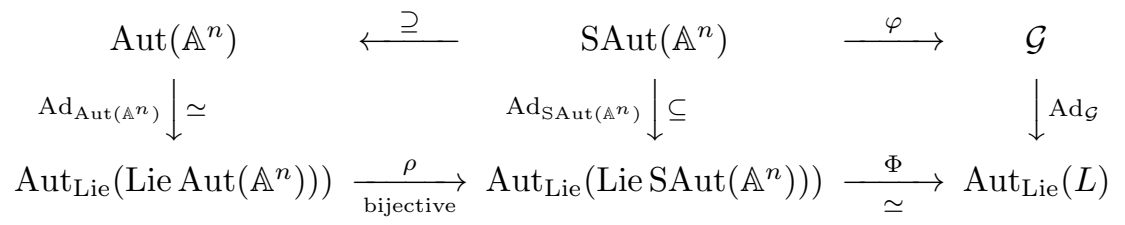

where $\operatorname{Ad}_{\operatorname{Aut}\left(\mathbb{A}^{n}\right)}$ is an isomorphism, by (1). Since $\rho$ is bijective ([KR17]) the composition $\rho \circ \operatorname{Ad}_{\operatorname{Aut}\left(\mathbb{A}^{n}\right)}$ is an isomorphism, again by (1). Therefore, the image $\mathfrak{A}:=$ $\operatorname{Ad}\left(\operatorname{SAut}\left(\mathbb{A}^{n}\right)\right) \subseteq \operatorname{Aut}_{\text {Lie }}\left(\operatorname{Lie} \operatorname{SAut}\left(\mathbb{A}^{n}\right)\right)$ is a closed subgroup isomorphic to $\operatorname{SAut}\left(\mathbb{A}^{n}\right)$, and $\mathfrak{A} \stackrel{\sim}{\longrightarrow} \Phi(\mathfrak{A})=\operatorname{Ad}_{\mathcal{G}}\left(\varphi\left(\operatorname{SAut}\left(\mathbb{A}^{n}\right)\right)\right.$. But $\varphi\left(\operatorname{SAut}\left(\mathbb{A}^{n}\right)\right) \subseteq \mathcal{G}$ is dense, and so 
$\operatorname{Ad}_{\mathcal{G}}(\mathcal{G})=\Phi(\mathfrak{A})$. Thus, the composition $\operatorname{Ad}_{\mathcal{G}} \circ \varphi: \operatorname{SAut}\left(\mathbb{A}^{n}\right) \rightarrow \Phi(\mathfrak{A})$ is an isomorphism, hence $\varphi$ is an isomorphism, by Proposition 4.1 below.

Proposition 4.1. Let $\mathcal{H}, \mathcal{G}$ be two ind-groups, and let $\varphi: \mathcal{H} \rightarrow \mathcal{G}, \psi: \mathcal{G} \rightarrow \mathcal{H}$ be two homomorphisms. If $\psi \circ \varphi=\mathrm{id}_{\mathcal{H}}$, then $\varphi$ is a closed immersion, i.e. $\varphi(\mathcal{H}) \subseteq \mathcal{G}$ is a closed subgroup and $\varphi$ induces an isomorphism $\mathcal{H} \stackrel{\sim}{\rightarrow} \varphi(\mathcal{H})$.

Proof. By base change we can assume that the base field $\mathbb{k}$ is uncountable. Let $\mathcal{H}=\bigcup_{i} \mathcal{H}_{i}$ and $\mathcal{G}=\bigcup_{j} \mathcal{G}_{j}$ where we can assume that $\mathcal{H}_{i} \subseteq \mathcal{G}_{i}$ for all $i$. Moreover, for every $i$ there is a $k=k(i)$ such that $\psi\left(\mathcal{G}_{i}\right) \subseteq \mathcal{H}_{k}$. By assumption, the composition $\psi \circ \varphi: \mathcal{H}_{i} \rightarrow \mathcal{G}_{i} \rightarrow \mathcal{H}_{k}$ is the closed embedding $\mathcal{H}_{i} \hookrightarrow \mathcal{H}_{k}$, hence the first map is a closed embedding. Thus $H_{i}:=\varphi\left(\mathcal{H}_{i}\right)$ is a closed subset of $\mathcal{G}_{i}$ and $H:=\varphi(\mathcal{H})=$ $\bigcup_{i} H_{i}$. Now the claim follows from Lemma 4.2 below by setting $S:=\operatorname{ker} \psi$.

Recall that a subset $S \subseteq \mathfrak{V}$ of an ind-variety $\mathfrak{V}$ is called ind-constructible if $S=\bigcup_{i} S_{i}$ where $S_{i} \subseteq S_{i+1}$ are constructible subsets of $\mathfrak{V}$.

Lemma 4.2. Let $\mathcal{G}$ be an ind-group, $H \subseteq \mathcal{G}$ a subgroup and $S \subseteq \mathcal{G}$ an indconstructible subset. Assume that $\mathbb{k}$ is uncountable and that

(1) $H=\bigcup_{i} H_{i}$ where $H_{i} \subseteq H_{i+1} \subseteq \mathcal{G}$ are closed algebraic subsets,

(2) the multiplication map $S \times H \rightarrow \mathcal{G}$ is bijective.

Then $H$ is a closed subgroup of $\mathcal{G}$.

Proof. Let $\mathcal{G}=\bigcup_{k} \mathcal{G}_{k}$. We have to show that for every $k$ there exists an $i=i(k)$ such that $H \cap \mathcal{G}_{k}=H_{i} \cap \mathcal{G}_{k}$. We can assume that $e \in S=\bigcup_{i} S_{i}$. Then, by assumption, $\mathcal{G}=\bigcup_{j} S_{j} H_{j}$. Since $S_{j} H_{j} \cap \mathcal{G}_{k}$ is a constructible subset of $\mathcal{G}_{k}$ it follows that there exists a $j=j(k)$ such that $\mathcal{G}_{k} \subseteq S_{j} H_{j}$ ([FK17, Lemma 1.6.4]). Setting $\dot{S}:=S \backslash\{e\}$ we get $\dot{S} H \cap H=\emptyset$. Thus, $\mathcal{G}_{k}=\left(\dot{S}_{i} H_{i} \cap \mathcal{G}_{k}\right) \cup\left(H_{i} \cap \mathcal{G}_{k}\right)$ and $H \cap \dot{S}_{i} H_{i}=\emptyset$, hence $H \cap \mathcal{G}_{k}=H_{i} \cap \mathcal{G}_{k}$.

Finally, we can prove Theorem 1.6.

Proof of Theorem 1.6. (1) We already know from Theorem 1.4 that an injective homomorphism $\varphi: \operatorname{Aut}\left(\mathbb{A}^{n}\right) \rightarrow \operatorname{Aut}\left(\mathbb{A}^{n}\right)$ is a closed immersion. We claim that $d \varphi_{e}: \operatorname{Lie} \operatorname{Aut}\left(\mathbb{A}^{n}\right) \rightarrow \operatorname{Lie} \operatorname{Aut}\left(\mathbb{A}^{n}\right)$ is an isomorphism. To show this, consider the linear action of $\mathrm{GL}_{n}(\mathbb{k})$ on Lie $\operatorname{Aut}\left(\mathbb{A}^{n}\right)$. We then have

$$
\operatorname{Lie} \operatorname{Aut}\left(\mathbb{A}^{n}\right) \subseteq \operatorname{Vec}\left(\mathbb{A}^{n}\right) \simeq \mathbb{k}^{n} \otimes \mathbb{k}\left[x_{1}, \ldots, x_{n}\right]=\bigoplus_{d} \mathbb{k}^{n} \otimes \mathbb{k}\left[x_{1}, \ldots, x_{n}\right]_{d}
$$

and the latter is multiplicity-free as a $\mathrm{GL}_{n}(\mathbb{k})$-module as well as an $\mathrm{SL}_{n}(\mathbb{k})$-module.

Now $\varphi\left(\mathrm{GL}_{n}(\mathbb{k})\right) \subseteq \operatorname{Aut}\left(\mathbb{A}^{n}\right)$ is a closed subgroup isomorphic to $\mathrm{GL}_{n}(\mathbb{k})$. Moreover, $d \varphi_{e}$ : Lie $\operatorname{Aut}\left(\mathbb{A}^{n}\right) \rightarrow \operatorname{Lie} \operatorname{Aut}\left(\mathbb{A}^{n}\right)$ is an injective linear map which is equivariant with respect to $\varphi: \mathrm{GL}_{n}(\mathbb{k}) \stackrel{\sim}{\rightarrow} \varphi\left(\mathrm{GL}_{n}(\mathbb{k})\right)$. Since $\varphi\left(\mathrm{GL}_{n}(\mathbb{k})\right)$ is conjugate to the standard $\mathrm{GL}_{n}(\mathbb{k}) \subseteq \operatorname{Aut}\left(\mathbb{A}^{n}\right)$ and since the representation of $\mathrm{GL}_{n}(\mathbb{k})$ on Lie Aut $\left(\mathbb{A}^{n}\right)$ is multiplicity-free, it follows that $d \varphi_{e}$ is an isomorphism. Thus $\mathcal{G}:=\varphi\left(\operatorname{Aut}\left(\mathbb{A}^{n}\right)\right) \subseteq \operatorname{Aut}\left(\mathbb{A}^{n}\right)$ is a closed subgroup with the same Lie algebra as $\operatorname{Aut}\left(\mathbb{A}^{n}\right)$, and we get the following commutative diagram (see proof of Theorem 1.4):

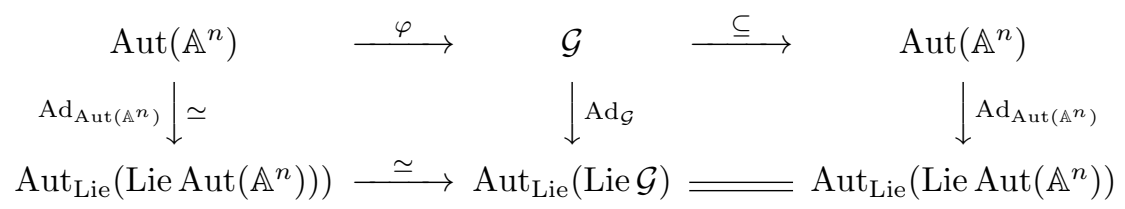


As a consequence, all maps are isomorphisms, and so $\mathcal{G}=\operatorname{Aut}\left(\mathbb{A}^{n}\right)$ and $\varphi$ is an isomorphism.

It remains to see that every automorphism $\varphi \in \operatorname{Aut}\left(\mathbb{A}^{n}\right)$ is inner. Since $\operatorname{Ad}$ is bijective (see $[\operatorname{KR} 17])$ and $d \varphi_{e} \in \operatorname{Aut}_{\text {Lie }}\left(\operatorname{Lie} \operatorname{Aut}\left(\mathbb{A}^{n}\right)\right)$ we get $d \varphi_{e}=\operatorname{Ad}(\mathbf{g})$ for some $\mathbf{g} \in \operatorname{Aut}\left(\mathbb{A}^{n}\right)$. This means that $d \varphi_{e}=(d \operatorname{Int} \mathbf{g})_{e}$ and so $\varphi=\operatorname{Int} \mathbf{g}$, by Proposition 2.7.

(2) The same argument as above shows that every nontrivial homomorphism $\operatorname{SAut}\left(\mathbb{A}^{n}\right) \rightarrow \operatorname{SAut}\left(\mathbb{A}^{n}\right)$ is an isomorphism where we use the fact that the action of $\mathrm{SL}_{n}(\mathbb{k})$ on Lie SAut $\left(\mathbb{A}^{n}\right)$ is multiplicity-free.

Moreover, $\operatorname{Ad}: \operatorname{Aut}\left(\mathbb{A}^{n}\right) \rightarrow \operatorname{Aut}_{\text {Lie }}\left(\operatorname{Lie} \operatorname{SAut}\left(\mathbb{A}^{n}\right)\right)$ is a bijective homomorphism of ind-groups, see [KR17]. Hence, for every $\varphi \in \operatorname{SAut}\left(\mathbb{A}^{n}\right)$ there is a $g \in \operatorname{Aut}\left(\mathbb{A}^{n}\right)$ such that $d \varphi_{e}=\operatorname{Ad} \mathbf{g}$ which implies that $\varphi=\operatorname{Int} \mathbf{g}$.

\section{A special subgroup of $\operatorname{Aut}(X)$, proof of Theorem 1.1}

Our Theorem 1.1 will follow from a more general result which we will describe now. For any affine variety $X$ consider the normal subgroup $\mathcal{U}(X)$ of $\operatorname{Aut}(X)$ generated by the unipotent elements of $\operatorname{Aut}(X)$, or, equivalently, by the closed algebraic subgroups of $\operatorname{Aut}(X)$ isomorphic to the additive group $\mathbb{k}^{+}$. This is an instance of a so-called connected group of automorphisms defined by RAMANUJAM in [Ram64]. The group $\mathcal{U}(X)$ defined above was introduced and studied in $\left[\mathrm{AFK}^{+} 13\right]$ where it is called the group of special automorphisms ${ }^{1}$ of $X$. In particular, they give a very interesting connection between transitivity properties of the group $\mathcal{U}(X)$ and the flexibility of the variety $X$.

We do not know if $\mathcal{U}(X) \subseteq \operatorname{Aut}(X)$ is closed, but we still have the notion of an algebraic subgroup $G \subseteq \mathcal{U}(X)$, namely a subgroup which is algebraic as a subgroup of $\operatorname{Aut}(X)$, see Remark 2.3. We will also need the notion of an "algebraic" homomorphism between these groups.

Definition 5.1. A homomorphism $\varphi: \mathcal{U}(X) \rightarrow \mathcal{U}(Y)$ is algebraic, if for any algebraic subgroup $U \subseteq \mathcal{U}(X)$ isomorphic to $\mathbb{k}^{+}$the image $\varphi(U) \subseteq \mathcal{U}(Y)$ is an algebraic subgroup and $\left.\varphi\right|_{U}: U \rightarrow \varphi(U)$ is a homomorphism of algebraic groups. We say that $\mathcal{U}(X)$ and $\mathcal{U}(Y)$ are algebraically isomorphic, $\mathcal{U}(X) \simeq \mathcal{U}(Y)$, if there exists a bijective homomorphism $\varphi: \mathcal{U}(X) \rightarrow \mathcal{U}(Y)$ such that $\varphi$ and $\varphi^{-1}$ are both algebraic.

Lemma 5.2. Let $\varphi: \mathcal{U}(X) \rightarrow \mathcal{U}(Y)$ be an algebraic homomorphism. Then, for any algebraic subgroup $G \subseteq \mathcal{U}(X)$ generated by unipotent elements the image $\varphi(G) \subseteq$ $\mathcal{U}(Y)$ is an algebraic subgroup and $\left.\varphi\right|_{G}: G \rightarrow \varphi(G)$ is a homomorphism of algebraic groups.

Proof. There exist closed subgroups $U_{1}, \ldots, U_{m} \subseteq G$ isomorphic to $\mathbb{k}^{+}$such that the multiplication map $\mu: U_{1} \times U_{2} \times \cdots \times U_{m} \rightarrow G$ is surjective. This gives the following commutative diagram

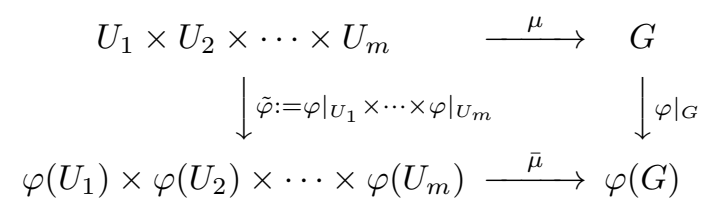

\footnotetext{
${ }^{1}$ They denote this group by $\operatorname{SAut}(X)$ which should not be confused with our definition of $\operatorname{SAut}\left(\mathbb{A}^{n}\right)$ and of SAut ${ }^{\text {alg }}(X)$ below.
} 
where all maps are surjective. It follows that $\overline{\varphi(G)} \subseteq \operatorname{Aut}(Y)$ is a (closed) algebraic subgroup, and thus $\varphi(G)=\overline{\varphi(G)}$, because $\varphi(G)$ is constructible. It remains to show that $\left.\varphi\right|_{G}$ is a morphism. This follows from the next lemma, because $G$ is normal, and $\mu$ and the composition $\left.\varphi\right|_{G} \circ \mu=\bar{\mu} \circ \tilde{\varphi}$ are both morphisms.

Lemma 5.3. Let $X, Y, Z$ be irreducible affine varieties where $Y$ is normal. Let $\mu: X \rightarrow Y$ be a surjective morphism and $\varphi: Y \rightarrow Z$ an arbitrary map. If the composition $\varphi \circ \mu$ is a morphism, then $\varphi$ is a morphism.

Proof. We have the following commutative diagram of maps

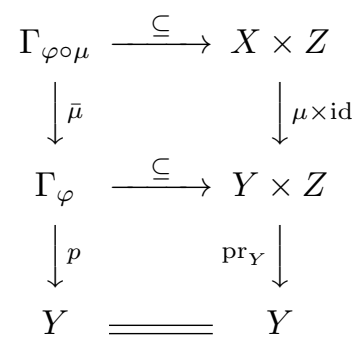

where $\Gamma_{\varphi \circ \mu}$ and $\Gamma_{\varphi}$ denote the graphs of the corresponding maps. We have to show that $\Gamma_{\varphi} \subseteq Y \times Z$ is closed and that $p$ is an isomorphism. The diagram shows that $\bar{\mu}$ is surjective, hence $\Gamma_{\varphi}$ is constructible, and $p$ is bijective. Thus, the induced morphism $\bar{p}: \overline{\Gamma_{\varphi}} \rightarrow Y$ is birational and surjective, hence an isomorphism since $Y$ is normal (see [Igu73, Lemma 4, page 379]). Since $p$ is bijective, we finally get $\Gamma_{\varphi}=\overline{\Gamma_{\varphi}}$.

Remark 5.4. If $\varphi: \operatorname{Aut}(X) \rightarrow \operatorname{Aut}(Y)$ is a homomorphism of ind-groups, then the induced homomorphism $\varphi_{\mathcal{U}}: \mathcal{U}(X) \rightarrow \mathcal{U}(Y)$ is algebraic. If $\operatorname{Aut}(X)$ and $\operatorname{Aut}(Y)$ are isomorphic as ind-groups, then $\mathcal{U}(X)$ and $\mathcal{U}(Y)$ are algebraically isomorphic.

The remark shows that the following result generalizes Theorem 1.1. The proof will be given in the next section.

Theorem 5.5. Let $X$ be a connected affine variety. If $\mathcal{U}(X)$ is algebraically isomorphic to $\mathcal{U}\left(\mathbb{A}^{n}\right)$, then $X$ is isomorphic to $\mathbb{A}^{n}$.

Finally, we define the following closed subgroups of $\operatorname{Aut}(X)$ :

$$
\begin{aligned}
\operatorname{Aut}^{\text {alg }}(X) & :=\overline{\langle G| G \subseteq \operatorname{Aut}(X) \text { connected algebraic }\rangle}, \\
\operatorname{SAut}^{a l g}(X) & :=\overline{\langle U| U \subseteq \operatorname{Aut}(X) \text { unipotent algebraic }\rangle .}
\end{aligned}
$$

We have $\operatorname{SAut}^{\text {alg }}(X)=\overline{\mathcal{U}(X)} \subseteq \operatorname{Aut}^{\text {alg }}(X) \subseteq \operatorname{Aut}(X)$. A similar argument as above gives the next result, again as a consequence of Theorem 5.5 above.

Corollary 5.6. Let $X$ be a connected affine variety. If $\mathrm{SAut}^{\text {alg }}(X)$ is isomorphic to SAut ${ }^{\text {alg }}\left(\mathbb{A}^{n}\right)$ as ind-groups, then $X$ is isomorphic to $\mathbb{A}^{n}$, and the same holds if we replace $\mathrm{SAut}^{\text {alg }}$ by $\mathrm{Aut}^{\text {alg }}$.

A special case of Theorem 1.1. Going back to our original Theorem 1.1 there is the following rather short proof in case $X$ is irreducible which was suggested by a referee. We first remark that the subgroup of translations $\mathcal{T} \subseteq \operatorname{Aut}\left(\mathbb{A}^{n}\right)$ is self-centralizing, i.e. $\operatorname{Cent}_{\mathrm{Aut}\left(\mathbb{A}^{n}\right)} \mathcal{T}=\mathcal{T}$. Denote by $\mathcal{T}^{\prime} \subseteq \operatorname{Aut}(X)$ the image of $\mathcal{T}$. We claim that $\mathcal{T}^{\prime}$ has a dense orbit. Since $\mathcal{T}^{\prime}$ is a unipotent group, this implies that $X$ is an orbit, hence isomorphic to $\mathbb{A}^{m}$ for some $m \leq n$. Since an $n$-dimensional torus acts faithfully on $X$, we have $n=m$, and we are done. 
It remains to see that $\mathcal{T}^{\prime}$ has a dense orbit in $X$, or equivalently, that every $\mathcal{T}^{\prime}$-invariant function on $X$ is a constant. Assume that this is not the case, and let $f \in \mathcal{O}(X)^{\mathcal{T}^{\prime}} \backslash \mathbb{k}$. Then we can "modify" every automorphism $\mathbf{t} \in \mathcal{T}$ by $f$ (see the following section 6 ) to obtain new unipotent automorphism $f \cdot \mathbf{t}$ in $\operatorname{Aut}(X)$ which do not belong to $\mathcal{T}^{\prime}$, but commute with $\mathcal{T}^{\prime}$, contradicting the fact that $\mathcal{T}^{\prime}$ is self-centralizing. (It is here where we use the irreducibility of $X$. Otherwise it is not clear why these modified automorphisms do not belong to $\mathcal{T}^{\prime}$.)

\section{Modifications and Root Subgroups, proof of Theorem 5.5}

Let $X$ be an affine variety and consider a nontrivial action of $\mathbb{k}^{+}$on $X$, given by $\lambda: \mathbb{k}^{+} \rightarrow \operatorname{Aut}(X)$. If $f \in \mathcal{O}(X)$ is $\mathbb{k}^{+}$-invariant, then we define the modification $f \cdot \lambda$ of the action $\lambda$ in the following way (see $\left[\mathrm{AFK}^{+} 13\right]$ where a modified action is called a replica; cf. [FK17, section 12.4]):

$$
(f \cdot \lambda)(s) x:=\lambda(f(x) s) x \text { for } s \in \mathbb{k} \text { and } x \in X .
$$

It is easy to see that this is again a $\mathbb{k}^{+}$-action. In fact, the action $\lambda$ corresponds to a locally nilpotent vector field $\delta_{\lambda} \in \operatorname{Vec}(X)$. Since $f$ is $\lambda$-invariant, it follows that $f \delta_{\lambda} \in \operatorname{Vec}(X)$ is again locally nilpotent, and defines the modified $\mathbb{k}^{+}$-action $f \cdot \lambda$. Note that if $U_{\lambda} \subseteq \operatorname{Aut}(X)$ denotes the image of $\lambda$, then $\operatorname{Lie}\left(U_{\lambda}\right) \stackrel{\sim}{\longrightarrow} \mathbb{k} \delta_{\lambda}$ under the canonical homomorphism Lie $\operatorname{Aut}(X) \hookrightarrow \operatorname{Vec}(X)$.

This modified action $f \cdot \lambda$ is trivial if and only if $f$ vanishes on every irreducible component $X_{i}$ of $X$ where the action $\lambda$ is nontrivial. It is clear that the orbits of $f \cdot \lambda$ are contained in the orbits of $\lambda$, and that they are equal on the open subset $X_{f}:=\{x \in X \mid f(x) \neq 0\}$ of $X$. In particular, if $X$ is irreducible and $f \neq 0$, then $\lambda$ and $f \cdot \lambda$ have the same invariants.

If $U \subseteq \operatorname{Aut}(X)$ is a closed subgroup isomorphic to $\mathbb{k}^{+}$and if $f \in \mathcal{O}(X)^{U}$ is a $U$ invariant, then we can define the modification $f \cdot U$ of $U$ by choosing an isomorphism $\lambda: \mathbb{k}^{+} \stackrel{\sim}{\longrightarrow} U$ and setting $f \cdot U:=(f \cdot \lambda)\left(\mathbb{k}^{+}\right)$, the image of the modified action.

Let $\mathcal{G}$ be an ind-group, and let $T \subseteq \mathcal{G}$ be a torus.

Definition 6.1. An algebraic subgroup $U \subseteq \mathcal{G}$ isomorphic to $\mathbb{k}^{+}$and normalized by $T$ is called a root subgroup with respect to $T$. The character of $T$ on Lie $U \simeq \mathbb{k}$ is called the weight of $U$.

If $U=U_{\lambda}$ is the image of a nontrivial $\mathbb{k}^{+}$-action $\lambda$, then $U$ is a root subgroup if and only if $\mathbb{k} \delta_{\lambda} \subseteq \operatorname{Vec}(X)$ is stable under $T$. If $\alpha$ is the weight of $U_{\lambda}$, we have

$$
t \cdot \lambda(s) \cdot t^{-1}=\lambda(\alpha(t) s) \text { for } t \in T, s \in \mathbb{k} .
$$

If a torus $T$ acts on an affine variety $X$, then we get a locally finite and rational representation of $T$ on the coordinate ring $\mathcal{O}(X)$, and thus a decomposition of $\mathcal{O}(X)$ into weight spaces. A locally finite and rational representation of $T$ is called multiplicity-free if the dimensions of the weight spaces are $\leq 1$. The following lemma is crucial.

Lemma 6.2. Let $X$ be an irreducible affine variety, and let $T \subseteq \operatorname{Aut}(X)$ be a torus. Assume that there exists a root subgroup $U \subseteq \operatorname{Aut}(X)$ with respect to $T$ such that $\mathcal{O}(X)^{U}$ is multiplicity-free. Then $\operatorname{dim} T \leq \operatorname{dim} X \leq \operatorname{dim} T+1$.

Proof. The first inequality $\operatorname{dim} T \leq \operatorname{dim} X$ is clear, because $T$ acts faithfully on $X$. It follows from [DK08, Propositions 2.7 and 2.9] that there exists a $T$-semi-invariant 
$f \in \mathcal{O}(X)^{U}$ such that the localization $\mathcal{O}(X)_{f}^{U}=\mathcal{O}\left(X_{f}\right)^{U}$ is finitely generated. Clearly, $\mathcal{O}(X)_{f}^{U}$ is $T$-stable and multiplicity-free, and $\mathcal{O}(X)_{f}^{U}$ is the coordinate ring of the algebraic quotient $Z:=X_{f} / / U$ on which $T$ acts. It follows from [Kra84, II.3.4 Satz 5]) that $T$ has a dense orbit in $Z$, and so $\operatorname{dim} Z \leq \operatorname{dim} T$. Since $\operatorname{dim} Z=$ $\operatorname{dim} X_{f} / / U=\operatorname{dim} X_{f}-1=\operatorname{dim} X-1$, we get the second inequality.

Lemma 6.3. We have $\mathcal{U}\left(\mathbb{A}^{n}\right) \subseteq \operatorname{SAut}\left(\mathbb{A}^{n}\right)$, and its closure $\overline{\mathcal{U}\left(\mathbb{A}^{n}\right)}$ is connected. Moreover, Lie $\overline{\mathcal{U}\left(\mathbb{A}^{n}\right)}=\operatorname{Lie} \operatorname{SAut}\left(\mathbb{A}^{n}\right)$, hence it is a simple Lie algebra.

Proof. The first statement is obvious, since every unipotent algebraic subgroup is contained in $\operatorname{SAut}\left(\mathbb{A}^{n}\right)$. The second claim follows from $\mathcal{U}\left(\mathbb{A}^{n}\right) \subseteq \overline{\mathcal{U}\left(\mathbb{A}^{n}\right)}$ ( (see Lemma 7.3 in the next section). For the last statement we remark that $\operatorname{Lie} \operatorname{SAut}\left(\mathbb{A}^{n}\right)$ is generated by the Lie algebras of the algebraic subgroups of $\operatorname{SAut}\left(\mathbb{A}^{n}\right)$ (Remark 2.6) which are all contained in $\mathcal{U}\left(\mathbb{A}^{n}\right)$ (Lemma 5.2).

Denote by $T_{n} \subseteq \mathrm{GL}_{n}(\mathbb{k}) \subseteq \operatorname{Aut}\left(\mathbb{A}^{n}\right)$ the diagonal torus and set $T_{n}^{\prime}:=T_{n} \cap \mathrm{SL}_{n}(\mathbb{k})$. The next result can be found in [Lie11, Theorem 1].

Lemma 6.4. Root subgroups of $\operatorname{Aut}\left(\mathbb{A}^{n}\right)$ with respect to $T_{n}^{\prime}$ exist, and their weights are all different.

Now we can give the proof of Theorem 5.5.

Proof of Theorem 5.5. The algebraic subgroups $\mathrm{SL}_{n}(\mathbb{k})$ and $\mathrm{SAff} n(\mathbb{k})$ of $\operatorname{Aut}\left(\mathbb{A}^{n}\right)$ both belong to $\mathcal{U}\left(\mathbb{A}^{n}\right)$ as well as all root subgroups $U$. Fix an algebraic isomorphism $\varphi: \mathcal{U}\left(\mathbb{A}^{n}\right) \stackrel{\sim}{\rightarrow} \mathcal{U}(X)$ and set by $T^{\prime}:=\varphi\left(T_{n}^{\prime}\right) \subseteq \mathcal{U}(X)$.

Let $X=\bigcup_{i} X_{i}$ be the decomposition into irreducible components. Since $\overline{\mathcal{U}(X)}$ is connected by Lemma 6.3 , the components $X_{i}$ are stable under $\overline{\mathcal{U}(X)}$. Denote by $A \subseteq$ $\mathcal{U}(X)$ the image of $\operatorname{Aff}_{n}(\mathbb{k})$ under $\varphi$. Since every nontrivial closed normal subgroup of $\operatorname{Aff}_{n}(\mathbb{k})$ contains the translations, one of the restriction maps $\rho_{i}: \mathcal{U}(X) \rightarrow \mathcal{U}\left(X_{i}\right)$, say $\rho_{1}$, is injective on $A$.

Let $T_{1}:=\rho_{1}\left(T^{\prime}\right) \subseteq \mathcal{U}\left(X_{1}\right)$ be the image of $T^{\prime}$. We will show that there is a root subgroup $U_{1} \subseteq \mathcal{U}\left(X_{1}\right)$ such that $\mathcal{O}\left(X_{1}\right)^{U_{1}}$ is multiplicity-free. Then Lemma 6.2 implies that $\operatorname{dim} X_{1} \leq n$ and so, by Proposition $2.8, X_{1}$ is isomorphic to $\mathbb{A}^{n}$ with a transitiv action of $A$. Since $X$ is connected, this implies that $X=X_{1} \simeq \mathbb{A}^{n}$.

In order to construct $U_{1}$ we choose a root subgroup $U \subseteq \varphi\left(\mathrm{SL}_{n}(\mathbb{k})\right) \subseteq \mathcal{U}(X)$, and set $U_{1}:=\rho_{1}(U) \subseteq \mathcal{U}\left(X_{1}\right)$. Since $U$ is a maximal unipotent subgroup of a closed subgroup $S \subseteq \mathcal{U}(X)$ isomorphic to $\mathrm{SL}_{2}(\mathbb{k})$ and since the restriction map res: $\mathcal{O}(X) \rightarrow \mathcal{O}\left(X_{1}\right)$ is a surjective homomorphism of $S$-modules, it follows that res: $\mathcal{O}(X)^{U} \rightarrow \mathcal{O}\left(X_{1}\right)^{U_{1}}$ is also surjective (see [Kra84, III.3.1, Bemerkung 2]). If $\alpha$ is the weight of $U$ and $U_{1}$ and if $f \in \mathcal{O}\left(X_{1}\right)^{U_{1}}$ is an invariant of weight $\beta$, then $f=\left.\tilde{f}\right|_{X_{1}}$ for an invariant $\tilde{f} \in \mathcal{O}(X)^{U}$ of weight $\beta$, and so $\tilde{f} \cdot U$ is a root subgroup of weight $\alpha+\beta$ with $\rho_{1}(\tilde{f} \cdot U)=f \cdot U_{1}$. Since the root subgroups of $\operatorname{Aut}(X)$ have different weights, it finally follows that $\mathcal{O}\left(X_{1}\right)^{U_{1}}$ is multiplicity-free.

\section{Finite dimensional automorphism groups}

It is well-known that for a smooth affine curve $C$ the automorphism group $\operatorname{Aut}(C)$ is finite except for $C \simeq \mathbb{k}, \mathbb{k}^{*}$. Theorem 1.2 implies that every finite group appears as automorphism group of a smooth affine curve. There also exist examples of smooth affine surfaces with a discrete non-finite automorphism group, see [FK17, 
Proposition 12.7.1]. Recall that an ind-group $\mathcal{G}=\bigcup_{k} \mathcal{G}_{k}$ is called discrete if $\mathcal{G}_{k}$ is finite for all $k$, or equivalently, if $\operatorname{Lie} \mathcal{G}=\{0\}$.

Definition 7.1. An ind-group $\mathcal{G}=\bigcup_{k} \mathcal{G}_{k}$ is called finite dimensional, $\operatorname{dim} \mathcal{G}<\infty$, if $\operatorname{dim} \mathcal{G}_{k}$ is bounded above. In this case we put $\operatorname{dim} \mathcal{G}:=\max _{k} \operatorname{dim} \mathcal{G}_{k}$.

Definition 7.2. For an ind-group $\mathcal{G}=\bigcup_{k} \mathcal{G}_{k}$ we define

$$
\mathcal{G}^{\circ}:=\bigcup_{k} \mathcal{G}_{k}^{\circ}
$$

where $\mathcal{G}_{k}^{\circ}$ denotes the connected component of $\mathcal{G}_{k}$ which contains $e \in \mathcal{G}$.

An ind-variety $\mathcal{V}$ is called curve-connected if for every $v, w \in \mathcal{V}$ there is an irreducible curve $D$ and a morphism $D \rightarrow \mathcal{V}$ whose image contains $v$ and $w$. This is equivalent to the condition that $\mathcal{V}$ admits a filtration with irreducible varieties (see [FK17, Lemma 1.6.3]). The following result can be found in [FK17, Lemma 2.2.2]).

Lemma 7.3. Let $\mathcal{G}=\bigcup_{k} \mathcal{G}_{k}$ be an ind-group.

(1) $\mathcal{G}^{\circ} \subseteq \mathcal{G}$ is a curve-connected open (and thus closed) normal subgroup of countable index. In particular, Lie $\mathcal{G}=$ Lie $\mathcal{G}^{\circ}$.

(2) We have $\operatorname{dim} \mathcal{G}<\infty$ if and only if $\mathcal{G}^{\circ} \subseteq \mathcal{G}$ is an algebraic group.

(3) We have $\operatorname{dim} \mathcal{G}<\infty$ if and only if $\operatorname{dim} \operatorname{Lie} \mathcal{G}<\infty$.

Example 7.4. (1) We have Aut $\left(\mathbb{k}^{*}\right) \simeq \mathbb{Z} / 2 \ltimes \mathbb{k}^{*}$, hence Aut $\left(\mathbb{k}^{*}\right)^{\circ} \simeq \mathbb{k}^{*}$. Similarly, $\operatorname{Aut}\left(\mathbb{k}^{* n}\right) \simeq \mathrm{GL}_{n}(\mathbb{Z}) \ltimes \mathbb{k}^{* n}$, and so $\operatorname{Aut}\left(\mathbb{k}^{* n}\right)^{\circ} \simeq \mathbb{k}^{* n}$.

(2) Let $C:=V\left(y^{2}-x^{3}\right) \subseteq \mathbb{k}^{2}$ be NeILE's parabola. Then Aut $(C)=\mathbb{k}^{*}$. In fact, every automorphism of $C$ defines an automorphism of the normalization $\mathbb{A}^{1}$ of $C$ fixing the origin. From this the claim follows immediately.

(3) Let $C$ be a smooth curve with trivial automorphism group, and consider the one dimensional variety $Y_{C}=\mathbb{A}^{1} \cup C$ where the two irreducible components meet in $\{0\} \in \mathbb{A}^{1}$. Then $\operatorname{Aut}\left(Y_{C}\right) \simeq \mathbb{k}^{*}$. Moreover, the disjoint union $Y_{C_{1}} \cup$ $Y_{C_{2}} \cup \cdots \cup Y_{C_{m}}$ with pairwise non-isomorphic curves $C_{i}$ has automorphism group $\mathbb{k}^{* m}$. We will show in section 8 that for every $n$ there is even an irreducible affine variety $X$ whose automorphism group $\operatorname{Aut}(X)$ is an $n$ dimensional torus.

Theorem 1.3 claims that if $\operatorname{dim} \operatorname{Aut}(X)$ is finite, then either $X \simeq \mathbb{A}^{1}$ or $\operatorname{Aut}(X)^{\circ}$ is a torus. This follows immediately from the next result.

Proposition 7.5. Let $X$ be a connected affine variety. If $X$ admits a nontrivial action of the additive group $\mathbb{k}^{+}$, then either $X \simeq \mathbb{A}^{1}$ or $\operatorname{dim} \operatorname{Aut}(X)=\infty$.

Proof. If $X$ contains a one-dimensional irreducible component $X_{i}$ with a nontrivial action of $\mathbb{k}^{+}$, then $X_{i}$ is an orbit under $\mathbb{k}^{+}$, hence $X=X_{i} \simeq \mathbb{A}^{1}$. Otherwise, $\mathbb{k}^{+}$ acts non-trivially on an irreducible component $X_{j}$ of dimension $\geq 2$. Denote by $U \subseteq \operatorname{Aut}(X)$ the image of $\mathbb{k}^{+}$. We claim that the modifications $f \cdot U$ for $f \in \mathcal{O}(X)^{U}$ form an infinite dimensional subgroup $\mathcal{O}(X)^{U} \cdot U \subseteq \operatorname{Aut}(X)$. This follows if we show that the image of $\mathcal{O}(X)^{U}$ in $\mathcal{O}\left(X_{j}\right)$ is infinite dimensional. For that we first remark that there is a nonzero $U$-invariant $f$ which vanishes on all $X_{k}$ for $k \neq j$, because the vanishing ideal is $U$-stable. This implies that $X_{f} \subseteq X_{j}$, and so

$$
\mathcal{O}(X)_{f}^{U}=\mathcal{O}\left(X_{f}\right)^{U}=\mathcal{O}\left(X_{j}\right)_{f}^{U}=\left(\left.\mathcal{O}(X)^{U}\right|_{X_{j}}\right)_{f} .
$$

Thus the image $\left.\mathcal{O}(X)^{U}\right|_{X_{j}} \subseteq \mathcal{O}\left(X_{j}\right)$ is infinite dimensional. 
The following result - a partial converse of the proposition above - is due to Arzhantsev-GắFullin.

Proposition 7.6 ([AG17]). Let $X$ be an affine variety which does not admit a nontrivial $\mathbb{k}^{+}$-action. Then Aut $(X)$ contains a unique maximal torus $T$. If the action of $T$ on $X$ is one-fix-pointed, then $\operatorname{Aut}(X)^{\circ}=T$ and $\operatorname{Aut}(X) / T$ is a finite group.

(A $T$-action on $X$ is called one-fix-pointed if there is a unique fixed point $x_{0} \in X$ and no other closed orbit.) The paper [AG17] contains many examples of such varieties, e.g. cones over projective varieties with a finite automorphism group, or the so-called trinomial hypersurfaces.

\section{An EXAmple With a torus as AUtomorphism group}

In Example 7.4 we have mentioned that NeILE's parabola $C:=V\left(y^{2}-x^{3}\right) \subseteq \mathbb{k}^{2}$ has an automorphism group isomorphic to $\mathbb{k}^{*}$, and we have given an example of a reducible curve with automorphism group isomorphic to $\mathbb{k}^{* m}$. We now construct an irreducible variety $X$ of dimension $d$ with $\operatorname{Aut}(X) \stackrel{\sim}{\rightarrow} \mathbb{k}^{* d}$.

Definition 8.1. A plane curve $C \subseteq \mathbb{k}^{2}$ given by an equation of the form $y^{m}-x^{n}=0$ where $n>m \geq 2$ and $m, n$ are relatively prime, is called a cuspidal curve. It has an isolated singularity in the origin 0 .

For the cuspidal curve $C_{m, n}$ with equation $y^{m}=x^{n}$ we have a canonical isomorphism $\mathbb{k}^{*} \stackrel{\sim}{\rightarrow} \operatorname{Aut}\left(C_{m, n}\right)$ given by the action $t(x, y):=\left(t^{m} x, t^{n} y\right)$. The induced representation on the tangent space $T_{0} C_{m, n}=\mathbb{k}^{2}$ has weights $m, n$. In particular, $C_{m, n}$ is isomorphic to $C_{m^{\prime}, n^{\prime}}$ if and only if $(m, n)=\left(m^{\prime}, n^{\prime}\right)$. Moreover, the normalization is given by the bijective morphism $\mu_{C_{m, n}}: \mathbb{A}^{1} \rightarrow C_{m, n}, s \mapsto\left(s^{m}, s^{n}\right)$.

Proposition 8.2. Let $X$ be a product of $d$ cuspidal curves which are pairwise non-isomorphic. Then $\operatorname{Aut}(X) \simeq \mathbb{k}^{* m}$.

Proof. (a) Let $X=C_{1} \times C_{2} \times \cdots \times C_{d}$ be such a product. We have a canonical injective homomorphism $\rho_{X}: \mathbb{k}^{* d} \hookrightarrow \operatorname{Aut}(X)$. The normalization of $X$ is given by the bijective morphism

$$
\eta:=\eta_{1} \times \cdots \times \eta_{d}: \mathbb{A}^{d} \rightarrow X
$$

where $\eta_{i}: \mathbb{A}^{1} \rightarrow C_{i}$ is the normalization of $C_{i}$. For $j=1, \ldots, d$ define

$$
\tilde{C}_{j}:=\left\{\left(0, \ldots, c_{j}, \ldots, 0\right) \mid c_{j} \in C_{j}\right\} \subseteq X,
$$

i.e. $\tilde{C}_{j}$ is the image of the $j$ th coordinate line $L_{j} \subseteq \mathbb{A}^{d}$ under the normalization $\eta$. Then we have

$$
A_{X}:=\bigcup_{j} \tilde{C}_{j}=\left\{x \in X \mid \operatorname{dim} T_{x} X \geq 2 d-1\right\} .
$$

Now let $Y=D_{1} \times D_{2} \times \cdots \times D_{d}$ be another product of non-isomorphic cuspidal curves, and define $\tilde{D}_{j}$ and $A_{Y}$ as above. It follows from the description of $A_{X}$ and $A_{Y}$ that every isomorphism $\mu: X \stackrel{\sim}{\longrightarrow} Y$ induces an isomorphism $A_{X} \stackrel{\sim}{\longrightarrow} A_{Y}$. Hence there is a permutation $\sigma$ of $\{1, \ldots, d\}$ such that $C_{i} \simeq \tilde{C}_{i} \simeq \mu\left(\tilde{C}_{i}\right)=\tilde{D}_{\sigma(i)} \simeq D_{\sigma(i)}$.

(b) Now define $X_{j}:=\left\{c=\left(c_{1}, \ldots, c_{d}\right) \in X \mid c_{j}=0\right\} \simeq \prod_{i \neq j} C_{i}$. Clearly, $X_{j}$ is the image of the $j$ th coordinate hyperplane $H_{j} \subseteq \mathbb{A}^{d}$ given by $x_{j}=0$ under the normalization $\eta: \mathbb{A}^{d} \rightarrow X$. Since the singular points of $X$ are given by $X_{\text {sing }}=$ $\bigcup_{j} X_{j}$, it follows that every automorphism $\varphi: X \stackrel{\sim}{\rightarrow} X$ permutes the irreducible 
components $X_{j}$ of $X_{\text {sing }}$. Now (a) implies that the $X_{j}$ are pairwise non-isomorphic, hence $\varphi\left(X_{j}\right)=X_{j}$.

(c) By induction, we can assume that $\rho_{X_{j}}: \mathbb{k}^{* d-1} \rightarrow \operatorname{Aut}\left(X_{j}\right)$ is an isomorphism, and so $\varphi_{X_{j}}$ is given by an element $t_{j} \in \mathbb{k}^{* d-1}$. Looking at the intersections $X_{j} \cap$ $X_{k}$ we see that there is a $t \in \mathbb{k}^{* d}$ such that $\left.\varphi\right|_{X_{\text {sing }}}$ is given by $t$. Therefore, the automorphism $\psi:=t^{-1} \circ \varphi \in \operatorname{Aut}(X)$ induces the identity on $X_{\text {sing }}$. It follows that the normalization $\tilde{\psi}: \mathbb{A}^{d} \stackrel{\sim}{\longrightarrow} \mathbb{A}^{d}$ fixes the coordinate hyperplanes $H_{j}$ pointwise which implies that $\tilde{\psi}$ is the identity. In fact, if $\tilde{\psi}=\left(f_{1}, \ldots, f_{d}\right), f_{i} \in \mathbb{k}\left[x_{1}, \ldots, x_{d}\right]$, then we get $x_{i} \mid f_{i}$, and the claim follows because all $f_{i}$ are irreducible (see e.g. [Jel91] for a more general result).

\section{REFERENCES}

[AG17] Ivan V. Arzhantsev and Sergey A. Gaüfullin, The automorphism group of a rigid affine variety, Math. Nachr. 290 (2017), no. 5-6, 662-671.

$\left[\mathrm{AFK}^{+}\right.$13] Ivan Arzhantsev, Hubert Flenner, Shulim Kaliman, Frank Kutzschebauch, and Mikhail Zaidenberg, Flexible varieties and automorphism groups, Duke Math. J. 162 (2013), no. $4,767-823$.

[BKY12] Alexei Belov-Kanel and Jie-Tai Yu, On the Zariski topology of automorphism groups of affine spaces and algebras, arXiv:1207.2045v1, 2012.

[BW00] Yuri Berest and George Wilson, Automorphisms and ideals of the Weyl algebra, Math. Ann. 318 (2000), no. 1, 127-147.

[DK08] Harm Derksen and Gregor Kemper, Computing invariants of algebraic groups in arbitrary characteristic, Adv. Math. 217 (2008), no. 5, 2089-2129.

[FK17] Jean-Philippe Furter and Hanspeter Kraft, On the geometry of automorphism groups of affine varieties, in preparation, 2017.

[Igu73] Jun-ichi Igusa, Geometry of absolutely admissible representations, Number theory, algebraic geometry and commutative algebra, in honor of Yasuo Akizuki, Kinokuniya, Tokyo, 1973, pp. 373-452.

[Jel91] Zbigniew Jelonek, Identity sets for polynomial automorphisms, J. Pure Appl. Algebra 76 (1991), no. 3, 333-337.

[Jel15] _ On the group of automorphisms of a quasi-affine variety, Math. Ann. 362 (2015), no. 1-2, 569-578.

[Kra84] Hanspeter Kraft, Geometrische Methoden in der Invariantentheorie, Aspects of Mathematics, D1, Friedr. Vieweg \& Sohn, Braunschweig, 1984.

[Kra16] Algebraic Transformation Groups: An Introduction, Mathematisches Institut, Universität Basel, http://kraftadmin.wixsite.com/hpkraft, 2016.

[KR17] Hanspeter Kraft and Andriy Regeta, Automorphisms of the Lie algebra of vector fields on affine $n$-space, J. Eur. Math. Soc. (JEMS) 19 (2017), no. 5, 1577-1588.

[KRZ17] Hanspeter Kraft, Andriy Regeta, and Susanna Zimmermann, Small affine $\mathrm{SL}_{\mathrm{n}}$ varieties, in preparation, 2017.

[Kum02] Shrawan Kumar, Kac-Moody groups, their flag varieties and representation theory, Progress in Mathematics, vol. 204, Birkhäuser Boston Inc., Boston, MA, 2002.

[Lie11] Alvaro Liendo, Roots of the affine Cremona group, Transform. Groups 16 (2011), no. 4, $1137-1142$.

[Ram64] Chakravarthi P. Ramanujam, A note on automorphism groups of algebraic varieties, Math. Ann. 156 (1964), 25-33.

[Reg17] Andriy Regeta, Characterization of $n$-dimensional normal affine $\mathrm{SL}_{\mathrm{n}}$-varieties, arXiv: 1702.01173 [math.AG].

[Sha66] Igor R. Shafarevich, On some infinite-dimensional groups, Rend. Mat. e Appl. (5) 25 (1966), no. 1-2, 208-212.

[Sha81] $\quad$ On some infinite-dimensional groups. II, Izv. Akad. Nauk SSSR Ser. Mat. 45 (1981), no. 1, 214-226, 240. 
Universität Basel, Departement Mathematik und Informatik

Spiegelgasse 1, CH-4051 Basel, Switzerland

E-mail address: Hanspeter.Kraft@unibas.ch 